This is a preprint author copy of the paper published in the Biophysical Chemistry, v. 153, pages 27 - 35 (2010).

The final published copy of the paper can be found at the Elsevier Web site http://www.elsevier.com/locate/biophyschem 


\title{
Molecular Dynamics Simulations of Local Anesthetic Articaine in a Lipid Bilayer
}

\author{
Enamul H. Mojumdar and Alexander P. Lyubartsev* \\ Division of Physical Chemistry, Department of Materials and Environmental Chemistry, \\ Stockholm University, SE-106 91 Stockholm, Sweden.
}

\begin{abstract}
In order to investigate structural and dynamical properties of local anesthetic articaine in a model lipid bilayer, a series of molecular dynamics simulations have been performed. Simulations were carried out for neutral and charged (protonated) forms of articaine inserted in fully hydrated dimyristoylphosphatidylcholine (DMPC) lipid bilayer. For comparison purpose, a fully hydrated DMPC bilayer without articaine was also simulated. The length of each simulation was 200 ns. Various properties of the lipid bilayer systems in the presence of both charged and uncharged forms of articaine taken at two different concentrations have been examined: membrane area per lipid, mass density distributions, order parameters, radial distribution functions, head group tilt, diffusion coefficients, electrostatic potential, etc, and compared with results of previous simulations of DMPC bilayer in presence of lidocaine. It was shown that addition of both charged and neutral forms of articaine causes increase of the dipole electrostatic potential in the membrane interior.
\end{abstract}

Keywords: lipid bilayers, articaine, molecular dynamics, anesthetics, membranes

\section{Introduction}

Local anesthetics (lidocaine, procaine, cocaine, etc) are indispensable part of medical treatment. They have been used for a long days in practical medicine to relieve pain in a particular area and thereby facilitate the surgical procedure. Articaine is a relatively new local anesthetic used now in dentistry in many countries [1]. It is an amide type local anesthetic, and 
instead of benzene ring it contains a thiophene ring which increases its liposolubility. Unlike other local anesthetics, articaine is exceptional in that it contains an additional ester group which is rapidly metabolized by plasma esterase to articainic acid [2]. As a result, its half life, about 20 minutes, is also very short comparing to other local anesthetic [3]. Thus it can rapidly be cleared from the systemic circulation through kidney, minimizing the side effects. Generally, articaine solution contains a mixture of neutral and charged (protonated) forms of articaine with a pKa value of 7.8 [1]. However inside the membrane the equilibrium may be strongly shifted towards the uncharged form as it the case of other ionizable anesthetics [4].

Local anesthetics are believed to remove or smooth pain by blocking of voltage gated $\mathrm{Na}^{+}$or $\mathrm{K}^{+}$channels which are responsible for generation of action potential in the nerve endings [5]. The exact molecular mechanism is however not clearly understood yet because there exist many anesthetic molecules having rather different structures but similar actions [6]. Several different mechanisms have been considered in the literature. A prevailing point of view is that local anesthetics interact with the protein of ion channels by direct binding and change their functioning $[7,8,9,10]$. Local anesthetics may also interfere with the phospholipids packing in the membrane which may affect protein structure and function [11, 12, 13]. Among other possible pathways changes of membrane fluidity [14], membrane hydration [15], lateral pressure profile $[16,17]$ and changes in the membrane dipole electrostatic potential [16] have been considered in the literature.

Molecular dynamics computer simulation may provide valuable complementary to experiments information about details of interactions between the molecules. However, despite enormous growth of the number of works on computer simulations of lipid bilayers (for example see recent reviews $[18,19,20,21])$, the works on computer modeling of anesthetics in lipid bilayers are rather scant. In papers [22, 23] molecular dynamics simulations of general anesthetic halotane in lipid membrane have been described, which demonstrated that anesthetic molecules are associated mostly with polar groups of lipids. The length of these simulations was limited to 4 ns. In recent paper [24] it was demonstrated that addition of local anesthetic benzocaine increases disorder in the membrane. In a series of works [25, 26, 27], behavior of local anesthetic lidocaine in a model membrane composed of dimyristoylphosphatidylcholine (DMPC, or 14:0/14:0 PC) lipids, as well as changes in the membrane which might be responsible for the anesthetic action were investigated. Among other observations, it was shown [26] that 
addition of both charged and neutral forms of lidocaine causes noticeable increase of the dipole electrostatic potential in the membrane interior, which may be responsible for blocking of voltage-gated ion channel and thus for the anesthetic action. It seems interesting to compare results obtained for lidocaine - lipid bilayer system with simulations of the same bilayer with other anesthetics, in order to figure out similar features in the behavior of local anesthetics which might be essential for understanding of mechanisms behind the anesthetic action.

To the best of our knowledge so far, there are no published results on molecular dynamics simulations of aritcaine in a lipid bilayer. Experimentally, on the molecular level behavior of articaine in a DSPC lipid bilayer was studied by solid-state NMR [28] and in POPS and SOPS liposomes by differential scanning calorimetry [29]. These studies demonstrated that addition of articaine decrease membrane melting temperature as well as transition entalphy.

The aims of this investigation are the following. First, various characteristics of the behavior of charged and neutral forms of articaine in a model lipid membrane, such as articaine preferential orientation and localization, hydration, hydrogen bond formation, diffusion, etc, will be examined. Second, effect of articaine on different membrane properties will be investigated. Special attention will be given to the effect of articaine on electrostatic potential across membrane, which was previously hypothesized [26] as a possible mechanism of the anesthetic action. Finally, behavior of articaine in the bilayer will be compared with the behavior of lidocaine which was studied in previous works $[25,26]$.

\section{Methodology}

\subsection{Molecular structures}

Five different simulations have been carried out. In each of them 128 DMPC lipids were arranged in a bilayer with 64 lipids in each leaflet, which were hydrated by 3655 water molecules. In addition to this, two of these systems also contained 12 either charged or uncharged articaine molecules, and two other systems contained 36 molecules of either charged or uncharged articaine. For systems with charged articaine, 12 or 36 chloride ions were added to provide electroneutrality. The fifth system contained only lipid molecules and water, the later simulation was carried our for the reference. Given the fact that clinical concentration of articaine solution is $4 \%$ [1] and 
high octanol/water partition coefficient of articaine (257 according to Song et al [28]), one can expect rather high presence of articaine in lipid membrane at physiological conditions. Also, results of work [28] indicate that a membrane cannot accomodate more than $40 \mathrm{~mol} \%$ of articaine. By these reasons we can believe that our simulations with 9.3 and $28 \mathrm{~mol} \%$ of articaine in bilayer represent concentrations which may exist at physiological conditions.

The molecular structures of DMPC lipids, as well as of the charged and uncharged articaine molecules are shown in Figure 1.
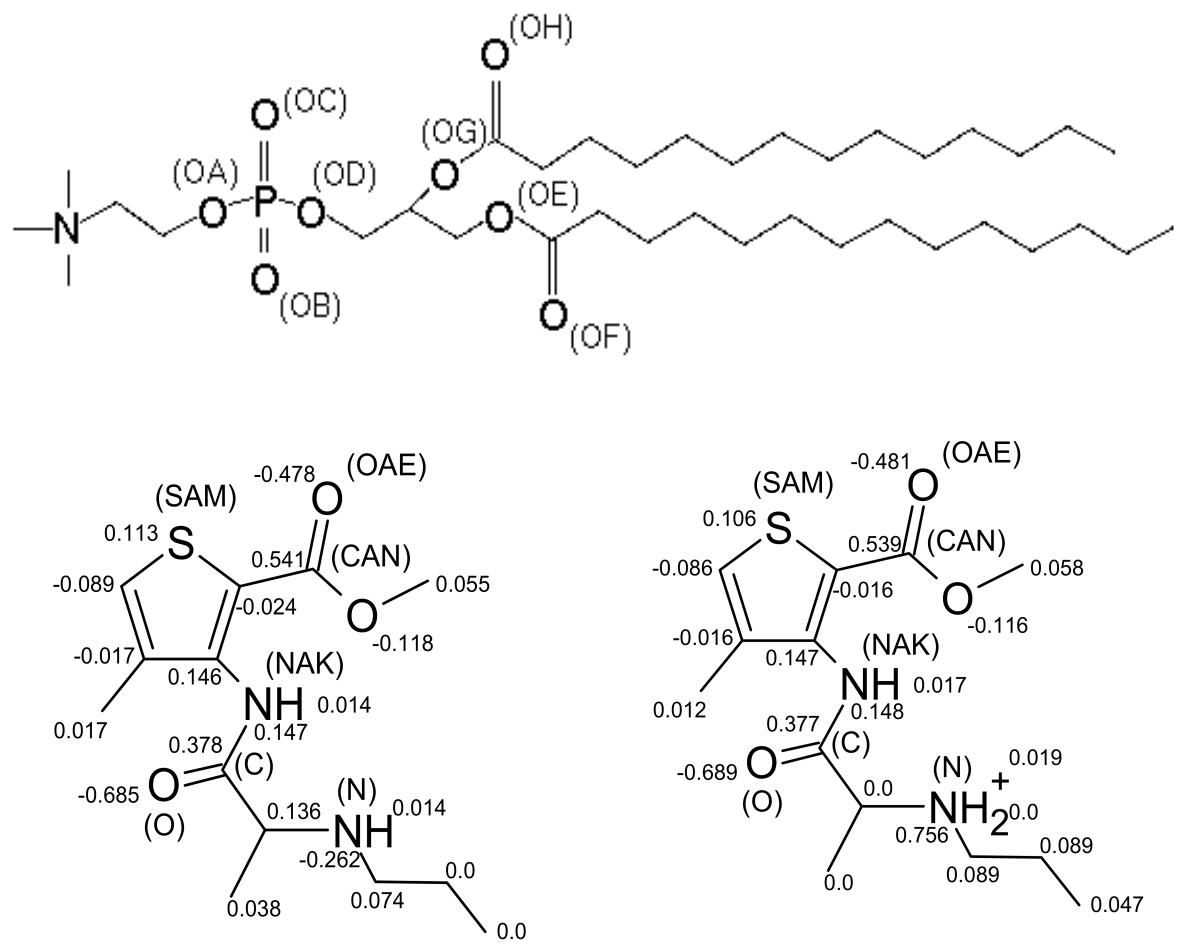

Figure 1: Molecular structure of DMPC lipid (top), neutral (bottom left) and charged (bottom right) forms of articaine, with partial atom charges and atom names referred in the text

For DMPC lipids, the united atom model based on the GROMOS [30] force field was used with modifications by Berger [31]. The force field was validated previously in works $[32,33,34]$. Also, the united atom approach was used to describe articaine drug molecules (except for the polar $\mathrm{H}$ atoms bound to the nitrogen atoms). For water molecules, the simple point charge (SPC) 
model was used [35]. The articaine topology files for the charged and uncharged forms with interaction parameters corresponding to the GROMACS "ffgmx" (known also as GROMOS87) force field were prepared using the PRODRG server [36] (available on-line at http://davapc1.bioch.dundee.ac.uk/prodrg/). The partial atom charges of the neutral and protonated forms of articaine are depicted in Fig.1.

\subsection{Simulation details}

During the simulations all the bonds were kept constrained with the LINCS algorithm [37] to remove fast vibrational motion. The time step was set to 2 femtosecond. The temperature was set to $310 \mathrm{~K}$ and was maintained using the Noose-Hoover thermostat scheme [38] with a coupling time constant of $0.1 \mathrm{ps}$. This temperature corresponds to the liquid crystalline structure of the neat DMPC bilayer. It was known from earlier studies that addition of local anesthetics decreases the temperature of the transition to the gel phase in lipid bilayers [39]. That is why the bilayer remains in the liquid crystalline phase upon addition of articaine molecules. The pressure was regulated by Parrinello-Rahman barostat [40] with a coupling time constant of $1.0 \mathrm{ps}$, which was applied semianisotropically with two degrees of freedom, one in the $\mathrm{Z}$ direction and another in the $\mathrm{XY}$ direction. The simulation box was allowed to extend in $\mathrm{Z}$ direction which is parallel to the bilayer normal and also in the XY plane throughout the simulation. The periodic boundary conditions were applied in all three directions.

The cutoff radius for Lennard-Jones interactions was set to $10 \AA$. For correct description of the bilayer structure, the long range electrostatic interactions are essential, and they have been maintained using Particle Mesh Ewald (PME) algorithm [41] with update every 10-th time step. Also, the long-range corrections of the Lennard-Jones interactions to the energy and pressure have been used. The eventual center of mass motion of the system was removed every 100 steps.

The initial structure for DMPC lipid bilayer (fully hydrated with 3655 water molecules) was taken from the biocomputing group at the University of Calgary (http://moose.bio.ucalgary.ca/). Then drug molecules were added outside the bilayer in equal amount from each side. Finally, ions and water have been added using tools included into GROMACS package [42]. Before the MD run, the systems were energy minimized using 1000 steps of the steepest descent method to remove any bad contacts and settle down the water molecules generated during setup. All five simulations were run 200 
ns from their initial conditions. All articaine molecules, dissolved initially in water, entered the bilayer after a few nanoseconds, which is consistent with a high octanol-water partition coefficient of articaine. The first $100 \mathrm{~ns}$ of the simulation were considered as equilibration and discarded while the last 100 ns were used for trajectory analysis. During the MD run, all coordinates were stored in the trajectories every 4 ps. The simulations, energy minimizations and some of analysis were conducted with the molecular dynamics package GROMACS v. 4.0.5 [42]. Analysis utilities from MDynaMix package [43] were used also. The simulations were run at the supercomputing cluster system at the Center for Parallel Computing, Stockholm, and proceeded at approximately 16 ns per day of wall clock time.

\section{Results and discussion}

\subsection{Area per lipid}

The average area per lipid is one of the most fundamental characteristics of lipid bilayers, as many other membrane properties like membrane thickness, elasticity, lateral diffusion, order parameters are related to it. The area per lipid was calculated in order to ensure the equilibration of the simulated systems. In this work, the average area per lipid molecule was calculated by multiplying the XY dimensions of the simulation box and divided by the number of lipid molecules present in one leaflet of the bilayer.

The time evolution of the area per lipid for different systems is presented in Figure 2. Over the last 100 ns of simulation there is no significant drift in evolution, and fluctuations of the area are typical to those observed in previous works $[32,34]$. Thus it can be concluded that all the systems were in equilibrium. The value of the area in the range $61-67 \AA^{2}$ corresponds to the liquid crystalline phase.

The average area per lipid for different systems is shown in Table 1, together with other important properties of lipid bilayer discussed further in the text. From the results, it can be seen that membrane in the presence of drug molecules has somewhat larger area per lipid than in the reference system. The reason is obvious: articaine molecules inserted into the membrane occupy some space which is responsible for increasing the average area. Simulation in the presence of uncharged articaine molecules shows slightly higher area per lipid (by $0.1 \AA^{2}$ ) than in the presence of charged articaine molecules though this difference is not statistically significant. Note that previous lidocaine simulation [26] showed larger effect (by about $1 \AA$ ) of the 


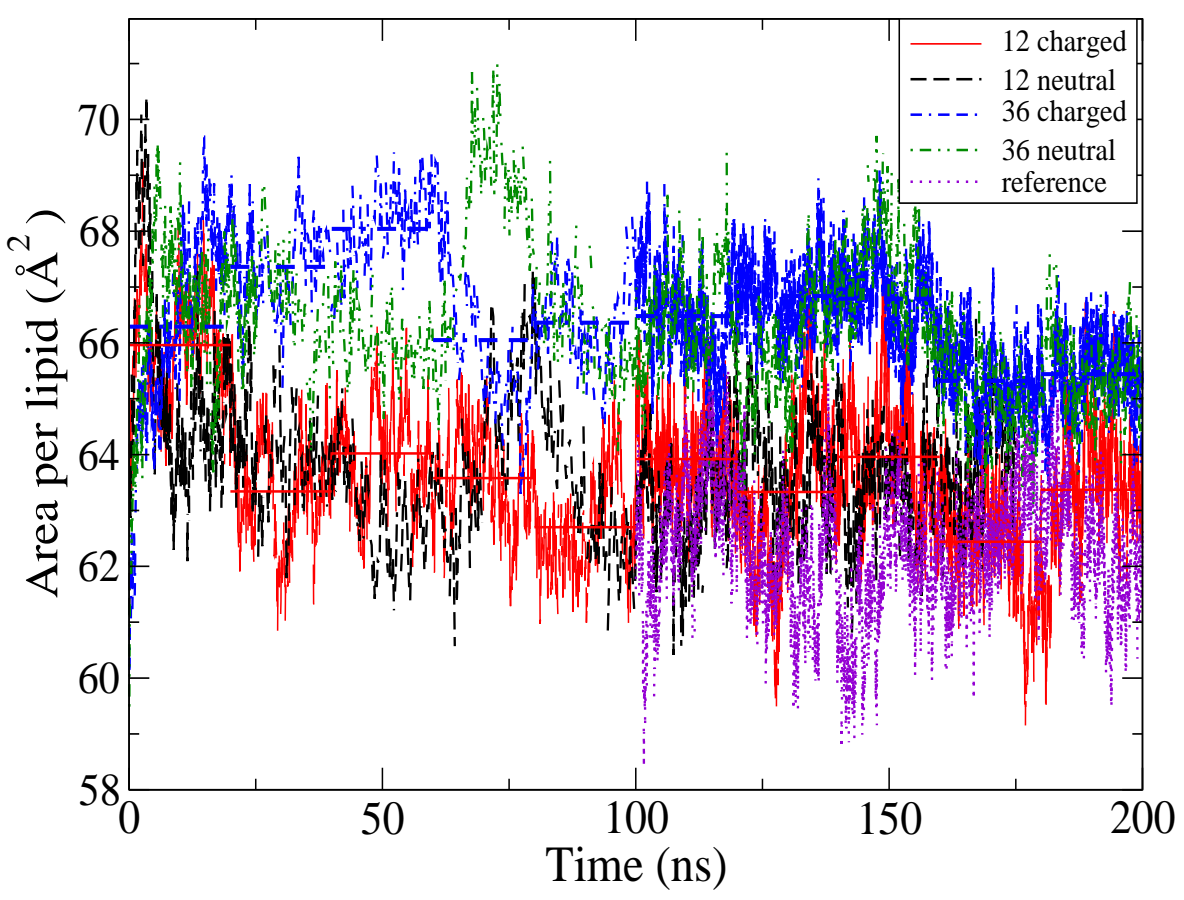

Figure 2: The time evolution of the area per lipid for the simulated systems. For charged systems, averages over $20 \mathrm{~ns}$ time intervals are shown as horizontal lines.

Table 1: Some important quantitative properties of the simulated bilayer system: $A$ - area per lipid; $h_{A}$ - distance from the articaine density maxima to the bilayer center; $S_{N-S A M}$ order parameter of N-SAM vector; $\alpha_{P N}$ - DMPC headgroup P-N tilt angle; $D_{A}$ - articaine lateral diffusion coefficient; $D_{L}$ - lipid lateral diffusion coefficient; $\psi_{\text {mid }}$ - electrostatic potential in the middle of membrane; $\psi_{\max }$ - maximum electrostatic potential. Error estimates are computed from the variance of averages computed from $10 \mathrm{~ns}$ pieces of the trajectory

\begin{tabular}{|l|cccccc|}
\hline System & $\begin{array}{c}12 \\
\text { charged }\end{array}$ & $\begin{array}{c}12 \\
\text { neutral }\end{array}$ & $\begin{array}{c}36 \\
\text { charged }\end{array}$ & $\begin{array}{c}36 \\
\text { neutral }\end{array}$ & $\begin{array}{c}\text { pure } \\
\text { bilayer }\end{array}$ & $\begin{array}{c}\text { error } \\
\text { estimate }\end{array}$ \\
\hline$A\left(\AA^{2}\right)$ & 63.6 & 63.7 & 64.9 & 65.0 & 63.0 & 0.5 \\
$h_{A}(\AA)$ & 12.1 & 8.9 & 13.1 & 9.5 & - & 0.4 \\
$S_{N-S A M}$ & -0.0793 & -0.129 & -0.0427 & -0.0024 & - & 0.05 \\
$\alpha_{P N}($ degree $)$ & 71 & 78 & 58 & 78 & 80 & 2 \\
$D_{A}\left(10^{-8} \mathrm{~cm}^{2} / s\right)$ & 4.2 & 9.1 & 3.6 & 16.1 & - & \\
$D_{L}\left(10^{-8} \mathrm{~cm}^{2} / s\right)$ & 5.7 & 8.9 & 4.0 & 14.4 & 9.7 & \\
$\psi_{\operatorname{mid}}(\mathrm{V})$ & 0.63 & 0.64 & 0.77 & 0.70 & 0.56 & 0.01 \\
$\psi_{\max }(\mathrm{V})$ & 0.81 & 0.76 & 0.92 & 0.74 & 0.77 & 0.02 \\
\hline
\end{tabular}


neutral lidocaine on the lipid area. For charged lidocaine, increase of the area relative to the reference bilayer was small [26], but for charged articaine increase of the area (comparing to the reference system) is about the same as for the neutral form. The reason for differences in the area per lipid for different systems might be due to the positioning and molecular orientation of articaine molecules in the membrane.

\subsection{Mass density}

The mass densities of the individual components for systems with both charged and uncharged forms of articaine, as well as for the reference bilayer are presented in Figure 3. The figure depicts that articaine molecules are located predominantly inside the bilayer under the lipid headgroups. Such behavior was observed previously for different anesthetics (halotane [23], lidocaine [25] and benzocaine [24]), as well as for alcohols [44, 45]. The two different forms of articaine prefer different depth in the bilayer. The charged articaine molecules have the density maximum at $13.1 \AA$ from the bilayer center in the area of lipid headgroup and esters, while neutral articaine molecules are located deeper in the bilayer, with the density maximum at $9.5 \AA$, which corresponds to the upper portion of the lipid tail region. Similar phenomena have been also seen for systems with 12 articaine molecules (data not shown).

The density for neutral articaine is not zero in the middle of bilayer, indicating that neutral molecules are able to cross the bilayer on the time scale of simulation. The dynamics of the bilayer crossing will be considered below, here we are pointing out that the computed density profile enables us to evaluate the free energy barrier for neutral articaine to cross the bilayer as $R T \ln \left(\rho_{\max } / \rho_{\text {mid }}\right)$ (where $\rho_{\max }$ and $\rho_{\text {mid }}$ are the maximum density of articaine and its density in the middle of bilayer, respectively). Computations with 12 neutral articaine molecules show the barrier of $9 \mathrm{~kJ} / \mathrm{mol}$ while in system with 36 drug molecules the barrier is $7 \mathrm{~kJ} / \mathrm{mol}$.

Another important observation is that DMPC lipids in the reference system have higher density maximum comparing to the charged and uncharged articaine systems. Position of this maximum is slightly shifted to the middle of bilayer for the charged system and to the bilayer surface for the uncharged system. An interesting feature is that charged lidocaine has its density maximum in the same (the most dense) area of the lipid bilayer. The effect of articaine on water distribution is rather small. Similar trends were also seen for lidocaine mass density distribution [25] 


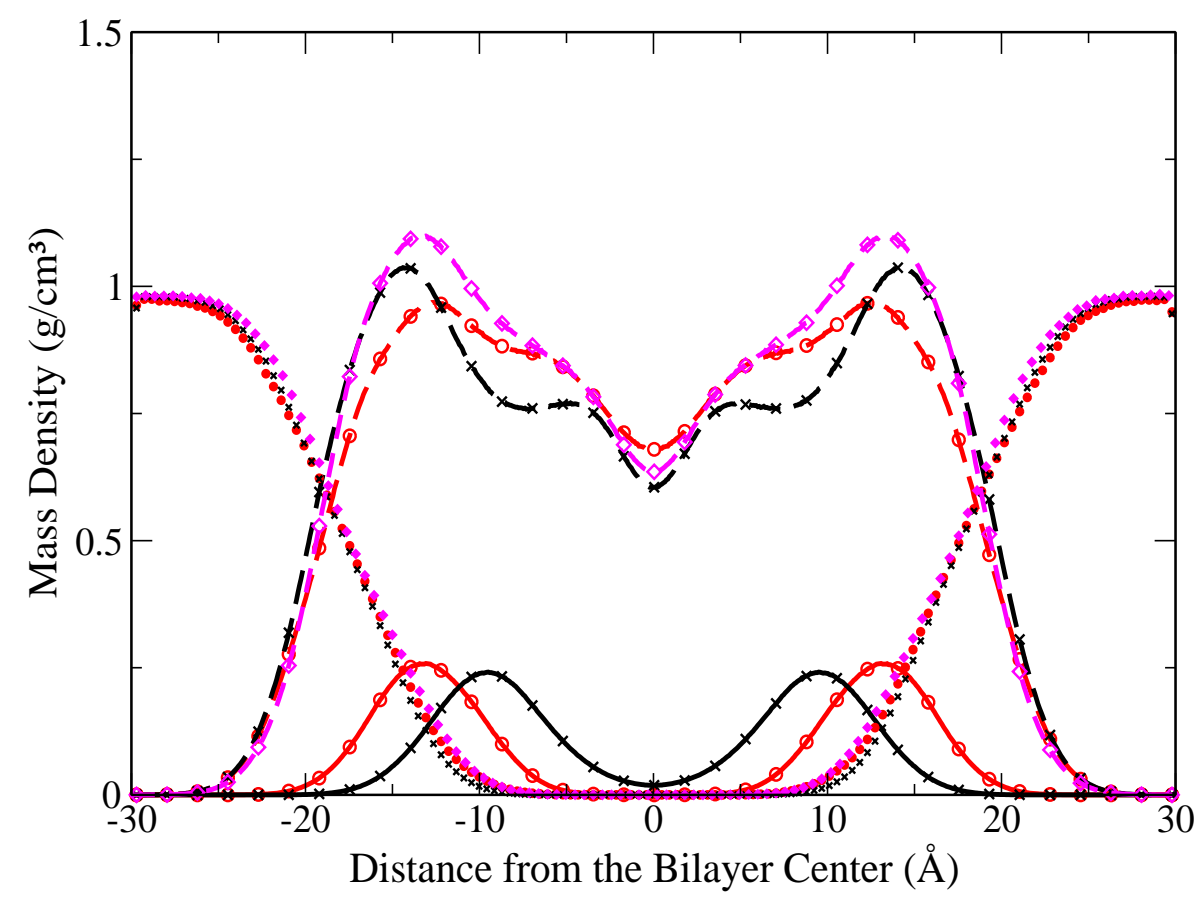

Figure 3: Mass density of different components as a function of distance from the bilayer center for 36 articaine molecules and for the reference bilayer. Charged articaine system: red lines with circles; uncharged articaine system: black lines with crosses; reference system: magenta lines with diamonds. Mass density of articaine: solid lines; DMPC: dashed lines; water: dotted lines. 


\subsection{Order parameters}

Order parameter is an important property describing orientation of molecules in anisotropic system. Order parameters related to $\mathrm{CH}$ bonds can be measured in NMR experiments. For a vector connected to a molecule, the order parameter is defined by:

$$
S=\frac{1}{2}\left\langle 3 \cos ^{2} \theta-1\right\rangle
$$

where $\theta$ is the angle between the molecular vector and Z-axis which is assumed to be parallel to the bilayer normal. Usually a molecular vector is defined by two chosen atoms. The vector $N-S A M$ (see Figure 1) can be associated with the articaine long axis, and we used this vector to define order parameter associated with orientation of articaine in the lipid bilayer.

The order parameters associated with the articaine long axis are given in Table 1. They show negative values for all cases, which means prevailing orientation perpendicular to the bilayer normal, or parallel to the bilayer surface. From the angular distribution of $N-S A M$ vector (Figure 4), as well as from visual inspection of trajectories it can be also seen that the molecules tend to be oriented perpendicular to the bilayer normal. This behavior differs from the behavior of lidocaine, for which the charged form adopts orientation essentially parallel to the bilayer normal [25]. The reason of such different behavior is that articaine molecule contains second carbonyl group which is partially polar. Thus the protonated articaine molecule has polar sites in the both ends of the molecule, which have larger affinity to the lipid headgroups than to membrane hydrophobic interior, and which provide orientation parallel to the membrane surface.

More information on orientation of articaine molecules can be obtained from the angular distribution of the articaine long axis associated with $\mathrm{N}-$ $S A M$ vector. These distributions are shown in Fig. 4. The zero angle corresponds to the direction out of bilayer. From the angular distribution, one can see noticeable differences in the orientation of charged and uncharged articaine. The charged system has the most probable angle of $49^{\circ}$ and $51^{\circ}$ for 12 and 36 systems respectively, and average angle of $68^{\circ}$ and $71^{\circ}$ degrees for the same systems. Also, charged systems have a second, but less pronounced, mode of orientation at angle about $110^{\circ}$. This indicates that the vector $N-S A M$ is directed most often out of the membrane, but in some cases it can reorient inward. On the contrary, the angular distribution of neutral 


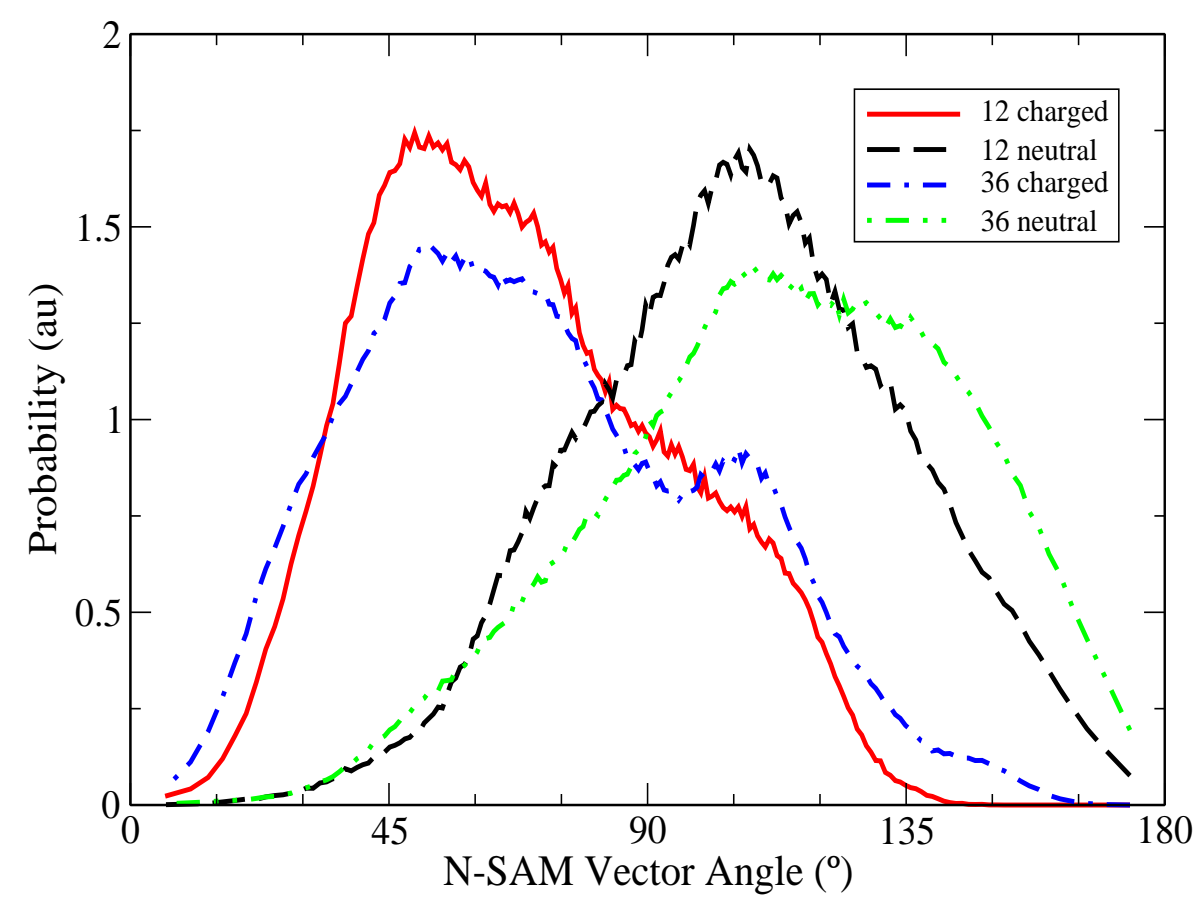

Figure 4: Angular distribution of the N-SAM vector of articaine molecules in the DMPC bilayer relative to the bilayer normal

articaine has the average angle of $108^{\circ}$ both for 12 and 36 systems, which indicates preferential inward pointing of the N-SAM vector in the bilayer.

Overall, it can be seen that articaine molecules adopt an orientation generally perpendicular to the bilayer normal in both charged and uncharged forms, whereas in the case of lidocaine only the uncharged form adopts the perpendicular orientation, the charged form being essentially parallel to the bilayer normal [25]. This also clarifies the difference in the behavior of the average area, discussed in the previous section. Since charged lidocaine is oriented parallel to the bilayer normal, it takes less space and increase average area per lipid less than charged articaine molecules.

Orientation and ordering of lipids in a bilayer is often described by $\mathrm{CH}$ (or deuterium) order parameters which are defined by directions of $\mathrm{CH}$ bonds belonging to different carbons of the chains. Such order parameter for carbons of $s n 1$ chain of lipids are shown in Figure 5 (calculations for the $s n 2$ chain show very similar result).

For the reference bilayer, the observed order parameters agree well with 


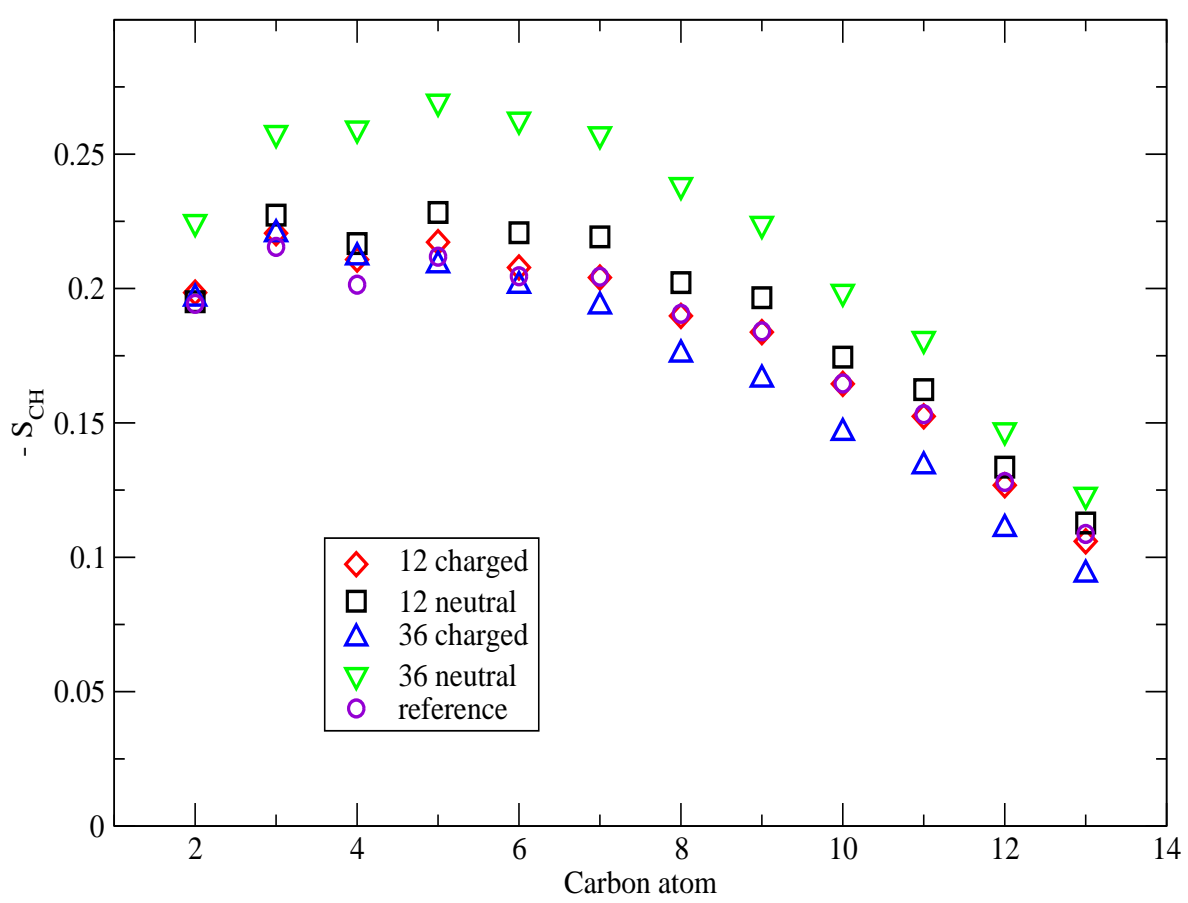

Figure 5: CH bond order parameters for different carbon atoms in the $s n 1$ chain of lipids 
previous simulations and experimental data (papers [34, 46] and references therein). The striking observation is that the neutral form of articaine causes noticeable increase of the order parameters. The apparent reason for this may be that uncharged lidocaine is located in the upper part of lipid tails, occupying certain space. Due to this reason, the density of lipids in this area is lower than in the reference case (see Fig. 3.), which means that the lipid chains goes straightly down to the middle of bilayer. Closer to the chain ends, the effect of neutral articaine on order parameters is weaker. For charged articaine, the effect is opposite: order parameters, especially closer to the end of the chain, becomes somewhat lower than those for the reference system

Previous studies showed that different inclusions into membrane may have different effect on the order parameters of the lipid tails. It is well known, both from simulations and experiments, that addition of cholesterol leads to increase of the order parameters [19]. Addition of local anesthetic benzocaine causes decrease of the order parameters, especially for phosphatidylserine lipids [24]. General anesthetic halotane does not show noticeable effect on the order parameters, while hexafluorethane increase the order parameters in saturated lipid chains [23]. Also the previous studies of DMPC membrane in presence of lidocaine showed increase of the order parameters, which was even qualitatively confirmed by the deuterium NMR [27]. Noteworthy, increase of the order parameters not necessarily means decrease of the membrane fluidity, since addition of neutral form of articaine lead to faster diffusion of lipids, as it will be discussed below.

\subsection{Head group tilt}

The head group tilt is a very important property of a phospholipid bilayer because the dipole moment associated with zwitterionic headgroup is involved in long-range electrostatic interactions affecting many properties of the bilayer [47]. Anesthetic molecules, located in the headgroup region, may exert influence of this angle as it was the case of charged lidocaine molecules studied in work [25]. Figure 6 depicts distribution of the head group P-N vector of DMPC lipids relative to the bilayer normal for each monolayer at different articaine concentrations as well as for the reference bilayer. The average angles between the $\mathrm{P}-\mathrm{N}$ vector and the bilayer normal are also given in Table 1.

According to the data presented in Table 1 and Figure 6, neutral articaine has small influence on the orientation of $\mathrm{P}-\mathrm{N}$ vector in the bilayer which is 


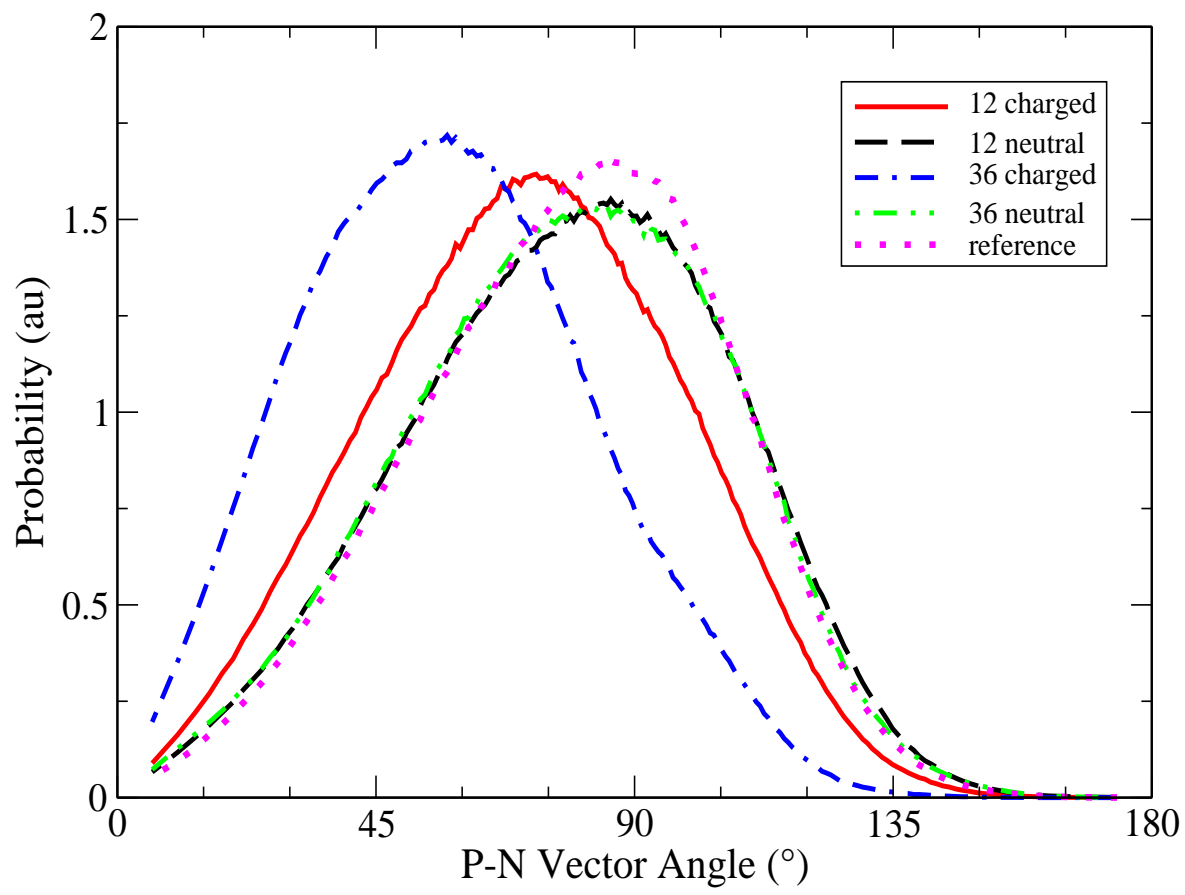

Figure 6: Distribution of the angle between P-N vector of DMPC head group and the outward normal of the bilayer 
almost the same (about $80^{\circ}$ ) as in the reference bilayer at both articaine concentrations. Even angular distribution of the vector remains essentially the same. Much more significant influence is observed in the case of charged articaine where for the system with 12 molecules the angle decreases to $71^{\circ}$, and further down to $58^{\circ}$ in the system with 36 charged articaine molecules. The reason for such strong influence of the charged articaine is that it is located predominantly in the area of lipid head groups, and, being positively charged, repel choline groups of lipids outwards to water. Neutral articaine molecules are mostly coordinated in the upper tail region of lipids rather than in the head group region, with no significant conformational changes of the P-N vector angle. Similar fashion of behavior of the PN vector was also observed in previous lidocaine simulations [26].

\subsection{Radial distribution functions}

Radial distribution functions (RDF) is one of the most important characteristics of the liquid structure. In order to get better insight into the coordination and hydration state of articaine and the probability distribution of water molecules around lipid and drug molecules, several RDF have been analyzed. The RDF of water oxygen around phosphate oxygen of the lipid head group, and RDF between two carbonyl oxygens of the lipid tail chains have been calculated in the presence of both charged and uncharged forms of articaine as well as also for the reference bilayer. They are presented in Figures 7,8 .

Figure 7 displaying the RDF of water oxygen around phosphate oxygen, shows similar behavior for all systems. All RDF have the first minimum at about $3.2 \AA$ which indicates the hydrogen bonding of water to phosphate oxygen, and also have a second maximum at around $5 \AA$. The charged sys-

tems have somewhat lower RDF comparing to the reference system, while RDF for neutral articaine systems are somewhat higher than RDF for the reference system. The effect becomes more noticeable for higher concentration with 36 articaine molecules. The coordination numbers, obtained from appropriate integration of RDF up to the first minimum, confirm the same trend: we obtained 1.77 for the reference system, 1.71 and 1.57 for the systems with 12 and 36 charged articaine molecules, and 1.78 and 1.79 for systems with 12 and 36 neutral articaine molecules respectively. This indicates that the charged systems have lower water distribution around the phosphate oxygens of lipids comparing to the reference system, while the uncharged systems have the opposite effect. This can be understood from 


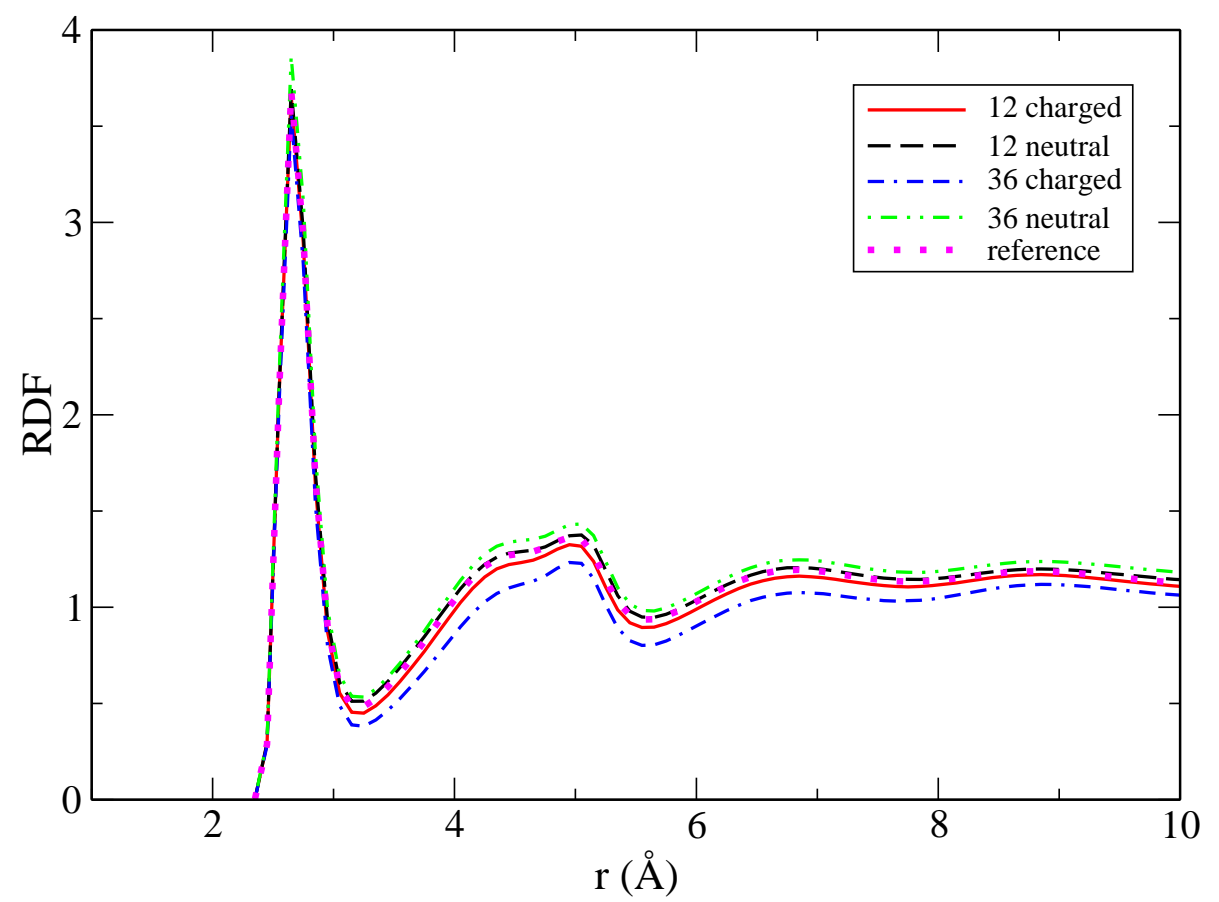

Figure 7: The radial distribution function of water oxygen around phosphate oxygen (OB and OC) of DMPC lipid molecules 


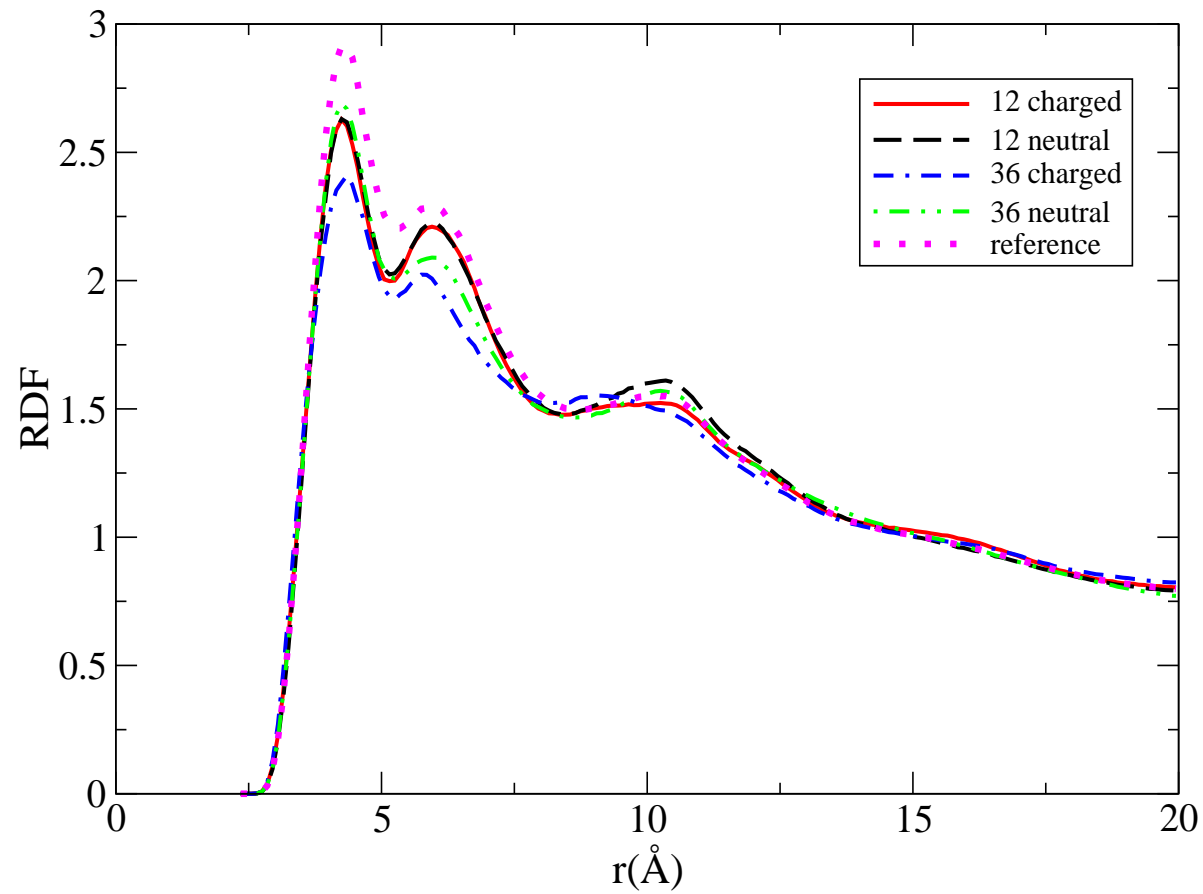

Figure 8: The radial distribution function between carbonyl oxygens of the two lipid tail chains $(\mathrm{OH}$ and $\mathrm{OF})$

the following considerations. From the mass density of articaine it is seen that the charged articaine molecules have a tendency to be located in the head group region and do not penetrate deeper into the bilayer. As a result, they compete with water molecules to form hydrogen bonds with DMPC phosphate oxygens and have the tendency to squeeze out water out of the bilayer. On the contrary, neutral articaine molecules are located in the upper part of the lipid tails and the ester group region i.e. closer to the bilayer center, leaving more space in the head group region, which can be filled by additional water molecules.

As the uncharged articaine molecules are located in the upper tail region, they push away the lipid tails belonging to different lipids. This results in decrease of the corresponding RDF between the lipid carbonyl groups comparing to the reference bilayer (see Figure 8). The charged systems also have lower RDF than the reference bilayer because of presence of the articaine molecules in the head group region, which propagate their effect up to the upper part of the lipid tail chains. The effect is pronounced better for the 
system with 36 molecules. Coordination numbers, computed for the first RDF minimum at $5.2 \AA$ and accounting for the carbonyl oxygens of the both lipid chains, confirm the same trend: they are 1.21 for the reference bilayer, 1.15 and 1.02 for systems with 12 and 36 charged articaine molecules, and 1.16 and 1.10 for systems with 12 and 36 neutral articaine molecules respectively.

\subsection{Hydrogen bonds}

Anesthetic molecules are able to form hydrogen bonds with water and different polar head group atoms of lipid and also compete with lipids for hydrogen bonds with water. This also was suggested as a possible mechanism for the anesthetic action of drug molecules [39]. Several hydrogen bonding patterns have been investigated: between articaine and lipid molecules, between articaine and water and also between lipids and water. The hydrogen bond was determined by the standard in Gromacs geometrical criteria (see e.g. [48]), with a maximum distance of $2.5 \AA$ between acceptor and hydrogen and a maximum angle of $30^{\circ}$ between the acceptor-hydrogen and hydrogendonor vectors. The results on statistics of different kinds of hydrogen bonds in the simulated systems are gathered in Table 2.

According to Table 2, charged articaine molecules typically form one hydrogen bond to DMPC while neutral articaine practically does not form hydrogen bonds to DMPC. It is also worth to note that most of such hydrogen bonds are formed between charged $\mathrm{NH}_{2}^{+}$group of articaine and phosphate oxygens of DMPC $(O B$ and $O C$, see Figure 1) followed by the carbonyl oxygens $(O F$ and $O H)$, ester oxygens $(O E$ and $O G)$, and phosphate oxygens $O A$ and $O D$.

The hydrogen bonding between articaine and water molecules also shows similar trends of being higher for charged articaine molecules comparing with uncharged one. Most of such hydrogen bonds are formed between carbonyl oxygen $(O A E)$ of the drug molecules and water. The hydrogen bonds with some other polar atoms like $N, N A K$ or $S A M$ were very few or virtually absent. The total amount of articaine-water hydrogen bonds per drug molecule is low even for the charged articaine.

The most interesting result of Table 2 is that the hydrogen bonding between DMPC and water shows strong effect (decrease) in case of charged articaine relative to the reference bilayer. The decrease of $\mathrm{DMPC}-\mathrm{H}_{2} \mathrm{O}$ hydrogen bonding is much stronger than increase of hydrogen bonding due to articaineDMPC and articaine-water interactions. Thus charged articaine has strong destroying influence on the hydrogen bond structure between DMPC and 
Table 2: Average number of different hydrogen bonds in the simulated systems

\begin{tabular}{|lll|}
\hline System & $\begin{array}{l}\text { Total bonds } \\
\text { per time frame }\end{array}$ & Bonds per articaine \\
\hline articaine - DMPC & & \\
12 neutral & 0.065 & 0.005 \\
12 charged & 12.25 & 1.021 \\
36 neutral & 0.250 & 0.007 \\
36 charged & 37.08 & 1.030 \\
& & \\
articaine $-\mathrm{H}_{2} \mathrm{O}$ & & \\
12 neutral & 0.210 & 0.018 \\
12 charged & 1.597 & 0.133 \\
36 neutral & 0.614 & 0.017 \\
36 charged & 5.758 & 0.16 \\
& & \\
DMPC - $\mathrm{H}_{2} \mathrm{O}$ & & bonds per DMPC \\
12 neutral & 710.048 & 5.54 \\
12 charged & 680.186 & 5.31 \\
36 neutral & 708.494 & 5.53 \\
36 charged & 609.250 & 4.76 \\
Reference & 714.301 & 5.58 \\
\hline
\end{tabular}


$\mathrm{H}_{2} \mathrm{O}$. This differs from the previously studied lidocaine behavior [25] where the effect of charged lidocaine on water-DMPC hydrogen bonding was indeed small. The probable reason for this behavior is that charged articaine is oriented predominantly parallel to the membrane surface in the lipid headgroup region, thus destroying DMPC-water hydrogen bonding, while charged lidocaine is oriented parallel to the bilayer normal.

\subsection{Lateral diffusion}

An important dynamical property of lipid bilayer systems is the diffusion. We calculated the lateral diffusion coefficients of articaine molecules as well as of the lipids. From the MD trajectories, the mean square displacement (MSD) of individual molecules was measured first and then the diffusion coefficient was calculated by the Einstein formula in two dimensions:

$$
D=\lim _{t \rightarrow \infty} \frac{1}{4} \frac{d}{d t}\left\langle\left[\vec{r}\left(t+t_{0}\right)-\vec{r}\left(t_{0}\right)\right]^{2}\right\rangle
$$

where $\vec{r}$ is a two-dimensional vector in the membrane plane defined by the center of mass of a molecule, $\langle\ldots\rangle$ indicates averaging over all possible initial times $t_{o}$ and molecules of the given type, and $D$ stands for the lateral diffusion coefficient. The brackets in eq. (2) define the time dependence of the lateral mean square displacement, which is shown in Figure 9. In practical calculations, the limit of the derivative in equation (2) is calculated from the slope of the linear region of the MSD vs. time curve. At shorter times (evaluated as 5 ns in ref. [46]) the MSD curve may show non-linear character due to non-Brownian character of the diffusion. At large times approaching to the total simulation time, MSD curves show typically large fluctuations due to poor statistics. In this calculation the slope was fit at the time interval 10-50 ns, where the MSD curves show behavior most close to linear, see Figure 9 .

The computed lateral diffusion coefficients for drug molecules and lipids are listed in Table 1. For the reference system, computed diffusion coefficient is well in line (taking into account temperature dependence) with previous simulations performed within the same force field $[34,46]$ as well as with experiment $[49,50]$. The lateral diffusion for charged articaine is almost twice slower comparing to the uncharged articaine for simulations with 12 drug molecules. The difference between charged and neutral forms becomes even larger at higher articaine concentration reaching factor about 5 for simulations with 36 drug molecules. The lateral diffusion of lipids changes also 


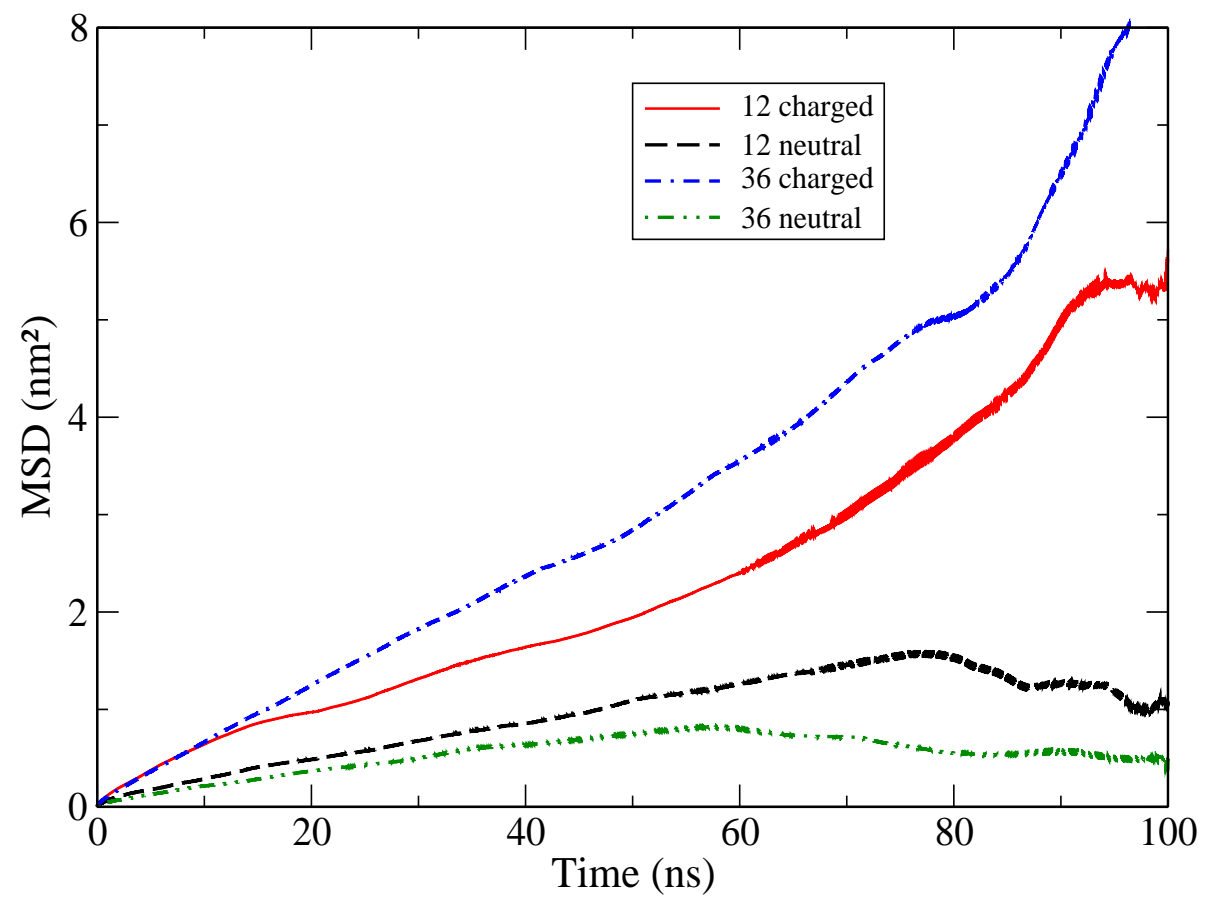

Figure 9: Lateral mean square displacement of articaine. 
with addition of articaine. The presence of charged articaine makes diffusion slower whereas neutral articaine makes it faster (some decrease of diffusion in the case of 12 neutral articaine may be not statistically significant). A qualitatively similar behavior was also observed in previous lidocaine simulation [25] but there are some quantitative differences. Comparison of data in Table 1 with results of work [25] shows that the lateral diffusion of articaine is somewhat slower comparing to the lidocaine diffusion.

\subsection{Crossing events}

Another important aspect of dynamics of articaine molecules in a lipid bilayer is their penetration through the middle of bilayer into another leaflet. The center of mass motion of drug molecules relative to the bilayer midplane, shown in Figures 10 a and b, was observed and analyzed for this purpose. One can see that charged molecules never cross the bilayer (Figure 10b) whereas uncharged molecules sometimes do so (Figure 10a). We have observed 7 completed transitions during 100 ns of observation. A transition was considered as completed if articaine molecule, after going through membrane mid-plane, remains in the other leaflet and not going immediately back. The observed rate corresponds to about one transition in 14 ns for 12 molecules, or in 170 ns for each individual articaine molecule.

Molecular trajectories in Figure 10 demonstrate also that not all crossing events were successful. Some molecules come across half way of the bilayer and went back within a few nanoseconds. Visualization of the transition pathway shows, that while crossing the middle part of the bilayer, articaine molecule stays essentially parallel to the bilayer normal, which is not its preferential orientation. If, after going to another side of the bilayer, the articaine molecule reorient itself parallel to the bilayer surface, it remains there for a longer time. Otherwise it can easily go back. Figure 10a shows 4 such unsuccessful crossing attempts. Similar trends were also found for 36 articaine systems (figure not shown).

\subsection{Electrostatic potential}

Change in the electrostatic potential inside membrane upon addition of anesthetic has been discussed as one of possible mechanisms of the anesthetic action $[16,26]$. The mechanism of a voltage gated ion channel can be affected by this potential. The membrane electrostatic potential arises mostly due to preferential orientation of the lipid head group dipoles and water dipoles at the membrane-water interface. That is why it is often referred to as bilayer 

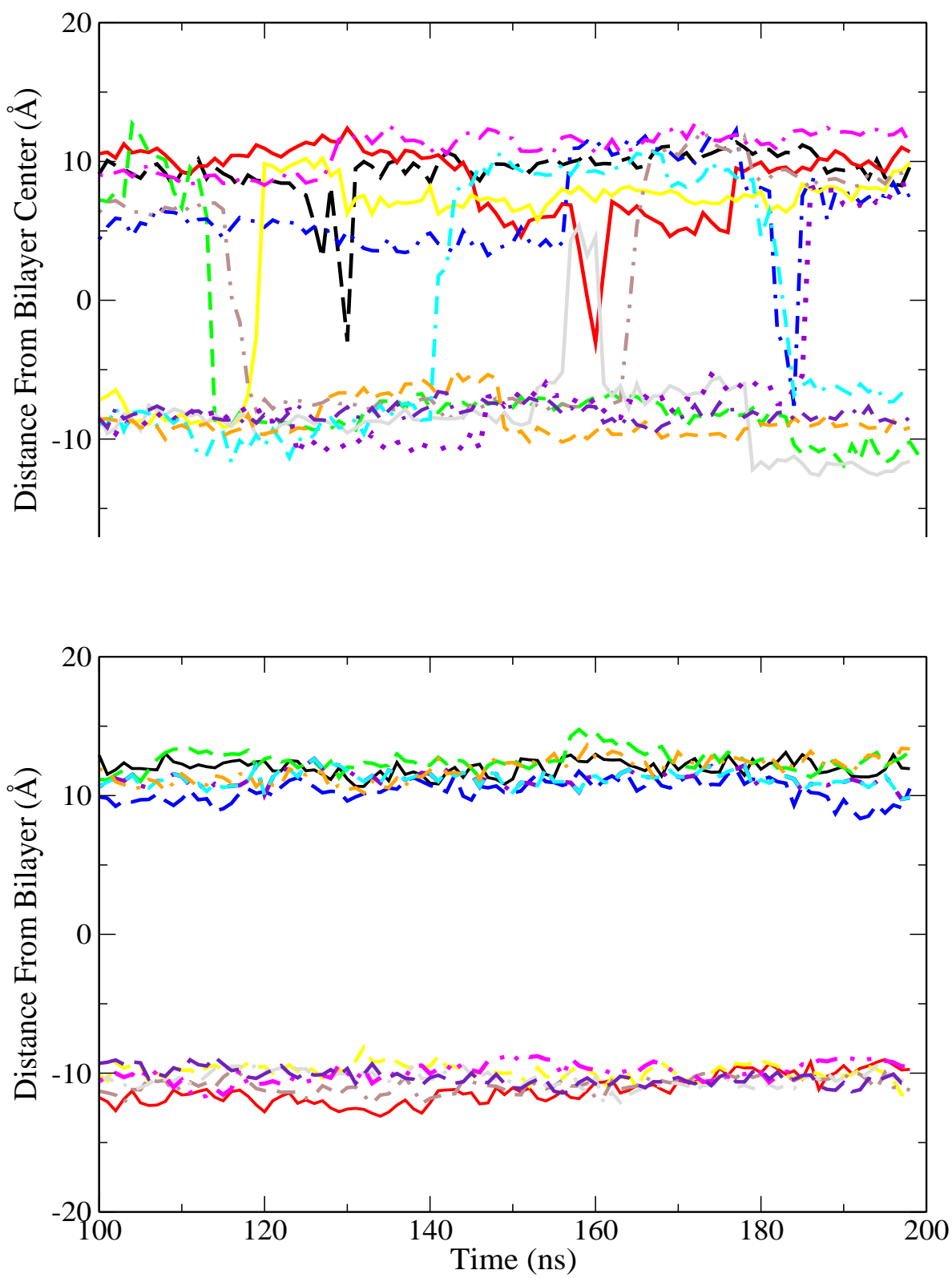

Figure 10: Distance from the bilayer center to the centers of mass of 12 uncharged (a) and 12 charged (b) articaine molecules as a function of time. Different molecules are represented by different colors and line styles. 
dipole potential. Other components (membrane associated molecules, ions) contribute also to the total electrostatic potential.

The electrostatic potential $\psi(z)$ across a lipid bilayer is linked with the charge density distribution along $\mathrm{Z}$ axis $\rho(z)$ via Poisson equation:

$$
\frac{d^{2} \psi(z)}{d z^{2}}=-\frac{\rho(z)}{\varepsilon_{0}}
$$

where $\varepsilon_{0}$ is the vacuum permittivity (the relative permittivity is set to 1 in atomistic simulations). The electrostatic potential is calculated by integrating equation (3) twice and using the boundary conditions for the potential and electric field to be zero at $z=z_{0}$ taken either at the center of the water layer or in the center of the membrane. We set zero potential at the center of the water layer $\left(z_{0}=-L_{z} / 2\right)$, then the equation for the potential becomes:

$$
\psi(z)=-\frac{1}{\varepsilon_{0}} \int_{z_{0}}^{z} d z^{\prime} \int_{z_{0}}^{z^{\prime}} \rho\left(z^{\prime \prime}\right) d z^{\prime \prime}
$$

The charge distribution $\rho(z)$ is computed from the partial atom charges defined in the force field. Before calculation of the potential according to eq. 4 , the charge density was symmetrized over both leaflets.

The total electrostatic potential of the simulated systems is presented in Figure 11. The values for the electrostatic potential in the middle of the membrane, as well as the maximum potential values are given in Table 1. For the reference system values of the potential are close to those observed in previous simulations using GROMOS force field $(500-600 \mathrm{mV}$ in the middle of membrane) $[44,47,26]$. Different experimental studies show different values of the dipole potential in the range of $200-800 \mathrm{mV}[51,52,53]$. Exact experimental determination of the dipole potential is tricky and may depend on interpretation of experimental observations, which explains different ranges of values given in different sources.

Figure 11 shows that both charge and uncharged articaine increase the electrostatic potential in the membrane interior. For the charged articaine, the potential increases by 70 and $210 \mathrm{mV}$ for systems with 12 and 36 drug molecules respectively. For neutral articaine, the increase is 80 and 140 $\mathrm{mV}$ for 12 and $36 \mathrm{drug}$ molecules respectively. It is remarkable that very similar rise of the electrostatic potential in the middle of the membrane was observed in previous lidocaine simulations [26]. The only noticeable difference from the lidocaine simulations, is that in the case of neutral articaine the 


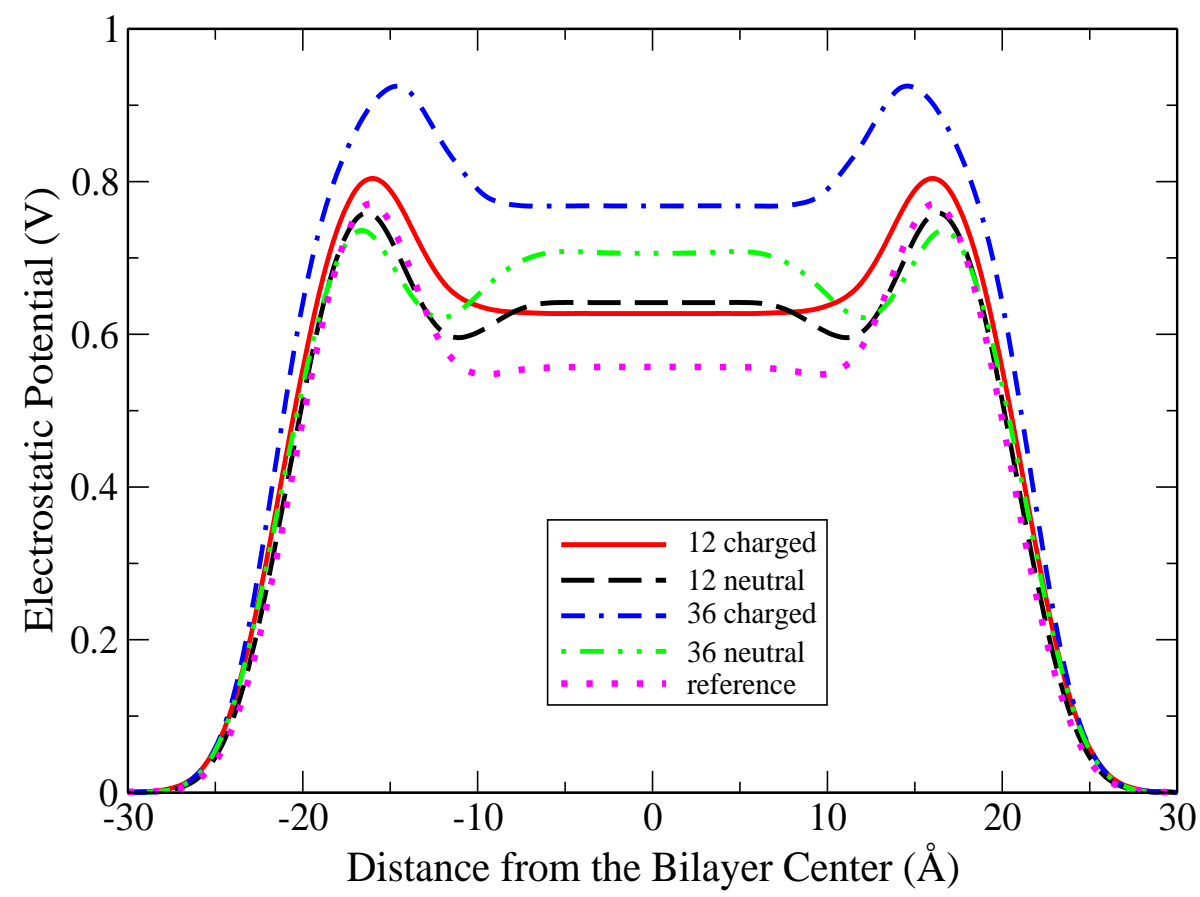

Figure 11: Membrane electrostatic potential 


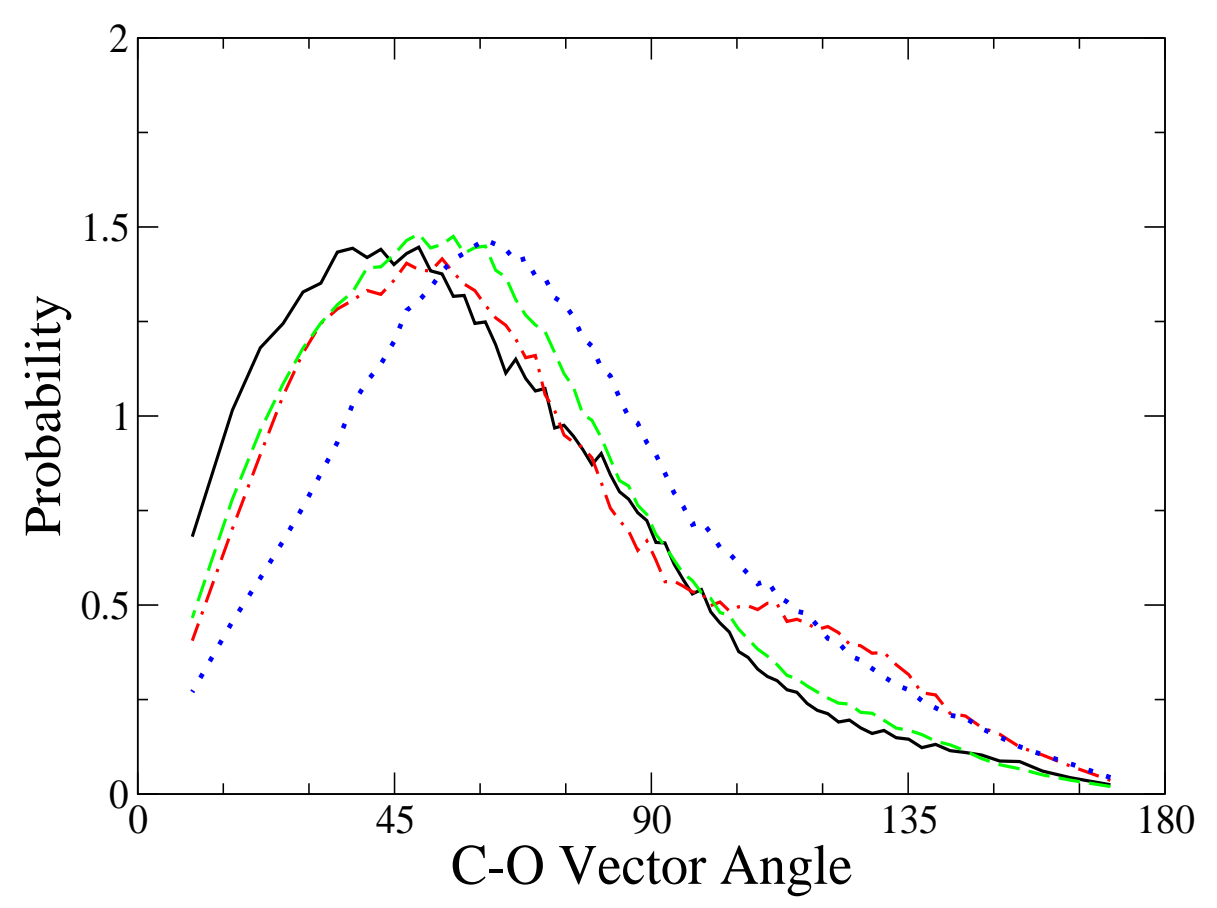

Figure 12: Probability distribution of the angle between outward direction of the bilayer normal and carbonyl bond C-O (black solid and green dashed lines) and CAN-OAE (red dash-dot and blue dot lines) of neutral articaine molecule. System with 12 articaine molecules: black solid and red dash-dot lines; system with 36 articaine molecules: green dashed and blue dot lines.

rise of the electrostatic potential seem to become slower at larger articaine concentrations.

While it is not surprisingly that positively charged drug molecules increase electrostatic potential inside bilayer, it less expected that their neutral forms do the same. The detailed analysis of different contributions to the electrostatic potential for bilayer with neutral lidocaine, made in paper [26], revealed that the main contribution to the increase of the electrostatic potential comes from the dipole moment of lidocaine itself. Our detailed investigation shows that the same mechanism works in the case of neutral articaine. While preferential orientation of the drug molecule is parallel to membrane surface, its carbonyl groups are oriented by oxygens directed out of membrane, as it can be seen from Figure 12 displaying probability distribution of the angle between both carbonyl $\mathrm{CO}$ bonds and outward direction of the membrane normal. The dipole moments associated with carbonyl groups 
contribute to the total increase of the electrostatic potential for systems with neutral articaine molecules. In the case of charged articaine, the resulting increase of the electrostatic potential is result of several contributions coming from the articaine ${ }^{+}-\mathrm{Cl}^{-}$double layer, change of the lipid headgroup tilt angle and reorientation of water dipoles, in the same manner as it was observed in previous lidocaine simulations [26].

\section{Conclusions}

We have analyzed different aspects of the behavior of both neutral and charged forms of articaine molecules in a DMPC lipid bilayer, as well as effect of articaine on membrane properties. From the results presented and discussed above, it can be concluded that the the charged and neutral forms of articaine show generally different behavior in the lipid bilayer. The charged articaine prefers the head group region of the bilayer (distantly located from the bilayer center) while neutral articaine prefers upper tail region of the bilayer (closer to the bilayer center). The neutral form increases bond order parameters in lipid tails while the charged form slightly decrease them. The both forms of articaine adopt an orientation perpendicular to the bilayer normal (parallel to the membrane surface). The charged articaine causes substantial decrease of the lipid headgroup tilt angle while the neutral form does not affect it. The charged articaine decreases hydration of the lipid headgroups and has destroying influence on hydrogen bonds between water and DMPC. The charged articaine makes diffusion slower but uncharged articaine makes it faster. The charged articaine never cross the bilayer whereas uncharged articaine sometimes does so.

The present investigation of articaine in lipid bilayer was carried out within the same methodological scheme (force field, simulation time, other simulation parameters) as previous lidocaine simulations $[25,26]$. While some properties of the two anesthetics were found to be very similar (location in the bilayer of the two forms, bilayer crossing, effect on the lipid headgroup angle), some other properties demonstrate differences: orientation of the charge form in the membrane, hydrogen bonding, diffusion. The major reason of differences in the behaviour of lidocaine and articaine can be traced to the presence of the second ester group in articaine which has high affinity to polar atoms of the lipid headgroup and water. As a result, charged articaine molecule adopt orientation parallel to the bilayer surface, which results also in 
stronger deterioration of the hydrogen bond structure comparing to charged lidocaine molecules.

The most interesting result of this study is that neutral articaine molecules increase the dipole electrostatic potential inside the membrane in almost the same manner as neutral lidocaine molecules, namely due to dipole moments of the carbonyl groups and their preferential orientation in the bilayer. From earlier works on molecular mechanisms of the anesthetic action [7] the prevailing point of view was that the charged form of local anesthetics is responsible for anesthetic action, due to its ability to bind to the receptor of ion channel $[9,54]$. On the other hand, due to difference in partition coefficients of the charged and uncharged forms, typically observed for ionizable local anesthetics, the neutral form should be predominant (at neutral $\mathrm{pH}$ ) in the membrane interior comparing to the charged form. The observed changes of the membrane dipole potential are of the order of typical values of the transmembrane potential, and thus may be a plausible reason of perturbing the normal functioning of voltage-gated ion channels. This issue is however still controversial because presence of membrane proteins, other types of phospholipids and other components also have influential roles. Further research will be necessary to investigate the behavior of these components inside membrane upon addition of anesthetic molecules.

\section{Acknowledgments}

This work has been supported by the Swedish Research Council (Vetenskapsrådet). Computer facilities have been granted by the Swedish National Infrastructure for Computing (SNIC).

\section{References}

[1] S. F. Malamed, S. Gagnon, D. Leblanc, Efficacy of articaine: A new amide local anesthetic, J. Am. Dent. Assoc. 131 (2000) 635 - 642.

[2] R. Oertel, R. Rahn, W. Kirch, Clinical pharmacokinetics of articaine, Clinical Pharmacokinetics 33 (1997) 417 - 425.

[3] S. P. V. Eeden, M. F. Patel, Prolonged paraesthesia following inferior alveolar nerve block using articaine, British Journal of Oral and Maxillofacial Surgery 40 (2002) $519-520$. 
[4] A. Avdeef, K. J. Box, J. E. A. Comer, C. Hibbert, K. Y. Tam, pHmetric LogP 10. Determination of liposomal membrane-water partition coefficients of ionizable drugs, Pharmaceutical Research 15 (1998) 209 -215 .

[5] J. F. Butterworth, G. R. Strichartz, Molecular mechanisms of local anesthesia: a review, Anesthesiology 72 (1990) 711 - 734 .

[6] R. G. Eckenhoff, Promiscuous ligands and attractive cavities: how do the inhaled anesthetics work, Mol Interv 1 (2001) 258 - 268.

[7] T. Narahashi, M. Yamada, D. T. Frazier, Cationic forms of local anaesthetics block action potentials from inside the nerve membrane, Nature 223 (1969) $748-749$.

[8] G. M. Lipkind, H. A. Fozzard, Molecular modeling of local anesthetic drug binding by voltage-gated sodium channels, Mol. Pharmacol. 68 (2005) $1611-1622$.

[9] G. K. Wang, J. Calderon, S. J. Jaw, S. Y. Wang, State-dependent block of $\mathrm{Na}+$ channels by articaine via the local anesthetic receptor, J. Membr. Biol. 229 (2009) 1 - 9.

[10] S. Vemparala, C. Domene, M. L. Klein, Computational studies on the interactions of inhalational anesthetics with proteins, Acc. Chem. Res. 132 (2010) $103-110$.

[11] Y. Boulanger, S. Schreier, I. C. Smith, Molecular details of anestheticlipid interaction as seen by deuterium and phosphorus-31 nuclear magnetic resonance, Biochemistry 20 (1981) $6824-6830$.

[12] M. Auger, I. C. Smith, H. H. Mantsch, P. T. Wong, High-pressure infrared study of phosphatidylserine bilayers and their interactions with the local anesthetic tetracaine, Biochemistry 29 (1990) 2008 - 2015.

[13] L. M. Pinto, D. K. Yokaichiya, L. F. Fraceto, E. de Paula, Interaction of benzocaine with model membranes, Biophys. Chem. 87 (2000) $213-$ 223.

[14] D. Papahadjoupoulos, K. Jacobson, G. Poste, G. Shepherd, Effect of local anesthetics on membrane properties. I. changes in the fluidity of phospholipid bilayer, Biochim. Biophys. Acta 394 (1975) $504-519$. 
[15] I. Ueda, T. Yoshiba, Hydration of lipid membranes and the action mechanisms of anesthetics and alcohols, Chem. Phys. Lipids 101 (1999) $65-79$.

[16] D. S. Cafiso, Dipole potentials and spontaneous curvature: membrane properties that could mediate anestesia, Toxicol. Lett. 100 - 101 (1998) $431-439$.

[17] R. S. Cantor, The influence of membrane lateral pressures on simple geometric models of protein conformational equilibria, Chem. Phys. Lipids 101 (1999) $45-56$.

[18] Y. H. M. Chan, S. G. Boxer, Model membrane systems and their applications, Curr. Opin. Chem. Biol. 11 (2007) 581 - 587.

[19] L. S. Vermeer, B. L. de Groot, V. Reat, A. Milon, J. Czaplicki, Acyl chain order parameter profiles in phospholipid bilayers: computation from molecular dynamics simulations and comparison with $2 \mathrm{H}$ NMR experiments, Eur. Biophys. J. 36 (2007) 919 - 931.

[20] S. J. Marrink, A. H. de Vries, D. P. Tieleman, Lipids on the move: Simulations of membrane pores, domains, stalks and curves, Biochim. Biophys. Acta 1788 (2009) 149 - 168.

[21] S. A. Pandit, H. L. Scott, Multiscale simulations of heterogeneous model membranes, Biochim. Biophys. Acta 1788 (2009) 136 - 148.

[22] K. Tu, M. Tarek, M. L. Klein, D. Scharf, Effects of anesthetics on the structure of a phospholipid bilayer: Molecular dynamics investigation of halothane in the hydrated liquid crystal phase of dipalmitoylphosphatidylcholine, Biophys. J. 75 (1998) 2123 - 2134.

[23] L. Koubi, L. Saiz, M. Tarek, D. Scharf, M. Klein, Influence of anesthetic and nonimmobilizer molecules on the physical properties of a polyunsaturated lipid bilayer, J. Phys. Chem. B 107 (2003) 14500 - 14508.

[24] R. D. Porasso, W. F. D. Bennett, S. D. Oliveira-Costa, J. J. L. Cascales, Study of the benzocaine transfer from aqueous solution to the interior of a biological membrane, J. Phys. Chem. B 113 (2009) 9988 - 9994. 
[25] C.-J. Högberg, A. Maliniak, A. P. Lyubartsev, Dynamical and structural properties of charged and uncharged lidocaine in a lipid bilayer, Biophys. Chem. 125 (2007) 416 - 424.

[26] C.-J. Högberg, A. P. Lyubartsev, Effect of local anesthetic lidocaine on electrostatic properties of a lipid bilayer, Biophys. J. 94 (2008) $525-$ 531.

[27] V. Castro, B. Stevensson, S. V. Dvinskikh, C.-J. Högberg, A. P. Lyubartsev, H. Zimmermann, D. Sandström, A. Maliniak, NMR investigations of interactions between anesthetics and lipid bilayers, Biochim. Biophys. Acta 1778 (2008) $2604-2611$.

[28] C. Song, H. Lygre, W. Nerdal, Articaine interaction with DSPC bilayer: A $13 \mathrm{C}$ and 31P solid-state NMR study, Eur. J. Pharm. Sci. 33 (2008) $399-408$.

[29] H. Lygre, G. Moe, W. Nerdal, H. Holmsen, Interaction of articaine hydrochloride with prokaryotic membrane, Acta Odontologica Scandinavica 67 (2009) $1-7$.

[30] D. P. Tieleman, H. J. C. Berendsen, Molecular dynamics simulations of a fully hydrated dipalmitoylphosphatidylcholine bilayer with different macroscopic boundary conditions and parameters, J. Chem. Phys. 105 (1996) $4871-4880$.

[31] O. Berger, O. Edholm, F. Jähnig, Molecular dynamics simulations of a fluid bilayer of dipalmitoylphosphatidylcholine at full hydration, constant pressure and constant temperature, Biophys. J. 72 (1997) 2002 2013.

[32] E. Lindahl, O. Edholm, Mesoscopic undulations and thickness fluctuations in lipid bilayers from molecular dynamics simulations, Biophys. J. 79 (2000) $426-433$.

[33] R. W. Benz, F. Castro-Roman, D. J. Tobias, S. H. White, Experimental validation of molecular dynamics simulations of lipid bilayers: A new approach, Biophys. J. 88 (2005) $805-817$.

[34] C.-J. Högberg, A. P. Lyubartsev, A molecular dynamics investigation of the influence of hydration and temperature on structural and dynamical 
properties of a dimyristoylphosphatidylcholine bilayer, J. Phys. Chem. B 110 (2006) 14326 - 14336.

[35] H. J. C. Berendsen, J. P. M. Postma, W. F. van Gunsteren, J. Hermans, Interaction models for water in relation to protein hydration, in: Intermolecular Forces, ed. by B. Pullman, D. Reidel Publishing Company, 1981, pp. $331-342$.

[36] A. W. Schüttelkopf, D. M. F. van Aalten, PRODRG: a tool for highthroughput crystallography of proteinligand complexes, Acta Crystallographica D 60 (2004) 1355 - 1363.

[37] B. Hess, H. Bekker, H. J. C. Berendsen, J. Fraaije, Lincs: A linear constraint solver for molecular simulations, J. Comput. Chem. 18 (1997) $1463-1472$.

[38] W. G. Hoover, Constant-pressure equations of motion, Phys. Rev. A 34 (1986) $2499-2500$.

[39] I. Ueda, J.-S. Chiou, P. R. Krishna, H. Kamaya, Local anesthetics destabilize lipid membranes by breaking hydration shell: infrared and calorimetry studies, Biochim. Biophys. Acta 1190 (1994) 421 - 429.

[40] M. Parrinello, A. Rahman, Crystal structure and pair potentials: A molecular-dynamics study, Phys. Rev. Lett. 45 (1980) 1196 - 1199.

[41] T. Darden, D. York, L. Pederson, Particle mesh Ewald: An $n \cdot \log (n)$ method for Ewald sums in large systems, J. Chem. Phys. 98 (1993) $10089-10092$.

[42] B. Hess, C. Kutzner, D. van der Spoel, E. Lindahl, Gromacs 4: Algorithms for highly efficient, load-balanced, and scalable molecular simulation, J. Chem. Theor. and Comput. 4 (2008) 435 - 447.

[43] A. P. Lyubartsev, A. Laaksonen, MDynaMix: a general and scalable parallel software package for arbitrary mixtures of molecules, Comput. Phys. Commun. 128 (2000) 565 - 589.

[44] M. Patra, E. Salonen, E. Terama, I. Vattulainen, R. Faller, B. W. Lee, J. Holopainen, M. Karttunen, Under the influence of alcohol: the effect of ethanol and methanol on lipid bilayers, Biophys. J. 90 (2006) 1121 1135. 
[45] U. R. Pedersen, G. H. Peters, P. Westh, Molecular packing in 1-hexanolDMPC bilayers studied by molecular dynamics simulations, Biophys. Chem. 125 (2007) $104-111$.

[46] J. Wohlert, O. Edholm, Dynamics in atomistic simulations of phospholipid membranes: Nuclear magnetic resonance relaxation rates and lateral diffusion, J. Chem. Phys. 125 (2006) 204703.

[47] J. Wohlert, O. Edholm, The range and shielding of dipole-dipole interactions in phospholipid bilayers, Biophys. J. 87 (2004) $2433-2445$.

[48] S. Y. Bhide, Z. Zhang, M. L. Berkowitz, Molecular dynamics simulations of SOPS and sphingomyelin bilayers containing cholesterol, Biophys. J. 92 (2007) $1284-1295$.

[49] P. F. F. Almeida, W. L. C. Vaz, T. E. Thompson, Lateral diffusion in the liquid phases of dimyristoylphosphatidylcholine/cholesterol lipid bilayers: A free volume analysis, Biochemistry 31 (1992) 6739 - 6747.

[50] G. Orädd, G. Lindblom, P. W. Westerman, Lateral diffusion of cholesterol and dimyristoylphosphatidylcholine in a lipid bilayer measured by pulsed field gradient NMR spectroscopy, Biophys. J. 83 (2002) 2702 2704 .

[51] G. Cevc, Membrane electrostatics, Biochim. Biophys. Acta 1031 (1990) $311-382$.

[52] R. J. Clarke, The dipole potential of phospholipid membranes and methods for its detection, Adv. Colloid Interface Sci. 89 - 90 (2001) 263 281.

[53] L. Wang, P. S. Bose, F. J. Sigworth, Using cryo-em to measure the dipole potental of a lipid membrane, Proc. Natl. Acad. Sci. USA 103 (2006) 18528 - 18533.

[54] C. A. Ahern, A. L. Eastwood, D. A. Dougherty, R. Hom, Electrostatic contribution of aromatic residues in the local anesthetic receptor of voltage-gated sodium channels, Circ. Res. 102 (2008) 86 - 94. 